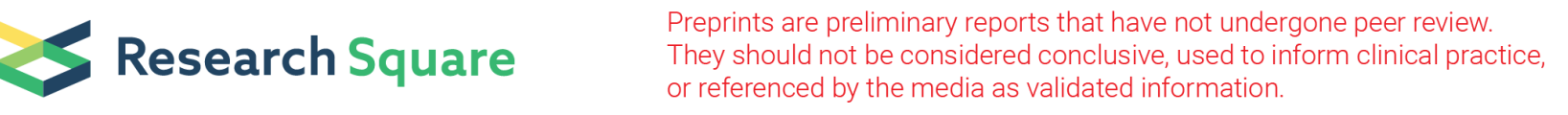

\title{
Triglyceride to HDL-C Ratio Is Associated with Plasma D- Dimer Levels in Different Types of Pancreatitis: A Retrospective Single-Centered Study in China
}

\section{Xiaoqing Jia}

Qilu Hospital of Shandong University

Xiaoting Zhang

Qilu Hospital of Shandong University

\section{Dalong Sun}

Qilu Hospital of Shandong University

Rong Li

Qilu Hospital of Shandong University

Na Yang

Qilu Hospital of Shandong University

Zheng Luo ( $\sim$ lizzyrichie@163.com )

Qilu Hospital of Shandong University

\section{Research Article}

Keywords: D-dimer, epidemiology, pancreatitis, dyslipidemia, coagulation

Posted Date: January 11th, 2022

DOI: https://doi.org/10.21203/rs.3.rs-1219746/v1

License: (c) (i) This work is licensed under a Creative Commons Attribution 4.0 International License. Read Full License 


\section{Abstract}

\section{Background}

This study aims to evaluate the relationship between D-dimer and dyslipidemia, especially triglyceride to HDL-C ratio (TG/HDL-C) in different types of pancreatitis. We analyzed the D-dimer and dyslipidemia levels in acute pancreatitis (AP), recurrent acute pancreatitis (RAP) and chronic pancreatitis (CP).

\section{Material and Methods}

A single-centered retrospective study was conducted on 1013 patients diagnosed with AP, RAP or CP. Only patients hospitalized within $24 \mathrm{~h}$ of onset were included, and 204 patients were enrolled in pancreatitis groups. 68 normal persons without pancreatitis, malignant diseases, pregnancy, or organ failure, who had health check-ups, were enrolled in the control group. Blood samples were taken within $24 \mathrm{~h}$ of admission. The epidemiology and etiology were analyzed. D-dimer and dyslipidemia levels were compared between different types of pancreatitis. Furthermore, the area under the receiveroperating characteristic curve (AUC) was used to estimate the validity of the predictor and to define optimal cut-off points for prediction.

\section{Results}

We found that D-dimer and TG/HDL-C ratio could distinguish mild AP (MAP) and non-MAP in AP and RAP patients. The Ddimer level was related to TG/HDL-C ratio and severity of pancreatitis, with the coefficient correlation of 0.379 and 0.427 ( $<<0.01$ ), respectively. TG/HDL-C was related to D-dimer in different types of pancreatitis. Multivariate analysis was conducted in the parameters at admission like alcohol abuse, dyslipidemia and coagulation disturbance in distinguishing AP and RAP groups from the control group, and the parameter like diabetes in RAP and CP groups significantly increased compared with that of the control group.

\section{Conclusions}

The value of D-dimer level and TG/HDL-C ratio in predicting the severity of AP and RAP was confirmed but there was no significant difference between CP group and the control group. The D-dimer level was related to dyslipidemia and TG/HDL-C ratio.

\section{Introduction}

Acute pancreatitis (AP) occurs due to the autodigestion of the pancreatic enzymes. It is an acute inflammatory process characterized by severe pain localized in the upper abdomen which may be radiating towards the back and can trigger a systemic inflammatory response [1]. Recurrent acute pancreatitis (RAP) is a clinical condition characterized by repeated episodes of acute pancreatitis, which is diagnosed retrospectively by clinical definition after at least the second episode of $\mathrm{AP}[2]$. RAP is an intermediary stage in the pathogenesis of chronic pancreatitis(CP), and a subset of RAP patients during their natural course transition to $\mathrm{CP}[3]$. CP involves progressive inflammatory and fibrotic changes of the exocrine pancreas, which could result in permanent structural damage and lead to impairment of both endocrine and exocrine functions[4]. It is known that the socioeconomic burden of pancreatitis is significant given the presence of symptoms and the costs to the health system [5]. The severe form accounting for $20-30 \%$ of acute pancreatitis patients is a life-threatening disease with high nosocomial mortality [6]. Therefore, identifying risk factors for RAP in patients with AP as early as possible may improve prevention and early treatment, thereby improving prognosis.

Pancreatitis induces venous thrombosis [7-9]. The pathogenesis of pancreatitis is release and activation of numerous proinflammatory cytokines leading to hypercoagulation and microvascular thrombosis, which may result in multi-organ dysfunction, splenic thrombosis and venous thromboembolism [10-14]. Thrombosis is a vascular complication of 
pancreatitis and a major cause of morbidity and mortality. Chronic pancreatitis accompanied by isolated thrombosis of the splenic vein is caused by perivenous inflammation of RAP[15].

D-dimer is the cleavage product of cross-linked fibrin that is formed by activation of the coagulation system, which signals hyperfibrinolysis in response to clot activation and fibrin formation[16]. D-dimer assays are commonly used in clinical practice to diagnose deep vein thrombosis or pulmonary embolism[17]. Recently, D-dimer levels are elevated in a variety of conditions, including coronary artery disease, infection and atrial fibrillation [18-20]. D-dimer is also found to be an early predictor of severity of acute pancreatitis [13]. However, the correlation between D-dimer levels and different types of pancreatitis is relatively unexplored.

Dyslipidemia include a low level of high-density lipoprotein cholesterol (HDL-C) and increased level of triglyceride (TG). The triglyceride to HDL-C ratio (TG/HDL-C) summarizes the combined effect of each level and can be used as a better predictor for insulin resistance, cardio-metabolic risk, and cardiovascular disease [21-23]. Besides, Several studies have reported that an elevated TG/HDL-C ratio correlated with the prevalence of CKD [24]. To our knowledge, few studies have evaluated TG/HDL-C ratios in different types of pancreatitis, and the relationship between TG/HDL-C and D-dimer has not been investigated.

In the present study, the plasma levels of D-dimer in patients with AP, RAP and CP were measured. Subsequently, correlations between D-dimer levels and different types of pancreatitis were assessed.

\section{Results}

\section{Patient and control characteristics}

Of 1013 patients with pancreatitis, 204 patients were enrolled and their serum D-dimer levels were all measured within $24 \mathrm{~h}$ of hospital admission. Baseline demographic and clinical characteristics were analyzed toward 95 patients with AP, 47 patients with RAP and 62 patients with CP (Fig. 1). Besides, 68 patients without pancreatitis, malignant diseases, pregnancy, or organ failure, who had regular physical check-ups, were enrolled in the control group. Of the 95 patients with AP, $40.00 \%$ were female and $60.00 \%$ were male, the median age was 50 . In the RAP group, $27.66 \%$ were female and $72.34 \%$ were male, the median age was 45 . Of the patients with CP, females were $20.97 \%$, and males were $79.03 \%$, the median age was 52.5 . In the control group, $33.82 \%$ were female and $66.18 \%$ were male, with a median age of 51 (Table 1). In AP patients, $35.79 \%$ had a smoking history, $41.05 \%$ had a history of drinking alcohol, $32.63 \%$ had hypertension, $9.47 \%$ had a history of cardiovascular disease (CVD), 13.68\% had type 2 diabetes, $20.00 \%$ had allergy history, 5.26\% had family chronic diseases, $23.16 \%$ had dyslipidemia and $70.53 \%$ had coagulation disorder. In RAP patients, $44.68 \%$ had a smoking history, $38.30 \%$ had a history of drinking alcohol, $14.89 \%$ had hypertension, $17.02 \%$ had a history of cardiovascular disease (CVD), $25.53 \%$ had type 2 diabetes, $17.02 \%$ had allergy history, $8.51 \%$ had family chronic diseases, $19.15 \%$ had dyslipidemia and $57.45 \%$ had coagulation disorder. In CP patients, $61.29 \%$ had a smoking history, $67.74 \%$ had a history of drinking alcohol, $30.65 \%$ had hypertension, $8.06 \%$ had a history of cardiovascular disease (CVD), 29.03\% had type 2 diabetes, 9.68\% had allergy history, $8.06 \%$ had family chronic diseases, $1.61 \%$ had dyslipidemia and $25.81 \%$ had coagulation disorder. Also, common etiologies, including biliary dysfunction, hypertriglyceridemia and alcoholism and idiopathic pancreatitis in different types of pancreatitis were shown in Table 2.

Gender, age, smoking status, alcohol drinking status, allergic history, presence of CVD, hypertension, type 2 diabetes, dyslipidemia, and coagulation disorder of these patients had been undergone multivariate analysis.

AP patients with coagulation disorder had an odds ratio (OR) of $13.88(95 \% \mathrm{Cl}, 6.22-30.98 ; \mathrm{P}=0.00)$ compared with patients in control group (Table 1). RAP patients with coagulation disorder had an OR of $12.53(95 \% \mathrm{Cl}, 5.51-28.49 ; \mathrm{P}=0.00)$ compared with patients in control group. AP patients with dyslipidemia had an OR of $8.40(95 \% \mathrm{Cl}, 4.11-17.15 ; \mathrm{P}=0.00)$ compared with patients in control group. RAP patients with dyslipidemia had an OR of $10.24(95 \% \mathrm{Cl}, 4.74-22.11 ; \mathrm{P}=0.00)$ 
compared with patients in control group. CP patients with dyslipidemia had an OR of $2.46(95 \% \mathrm{Cl}, 1.16-5.19 ; \mathrm{P}=0.02)$. CP patients with coagulation disorder had no significant difference compared with control group. AP patients with alcohol abuse had an OR of $6.74(95 \% \mathrm{Cl}, 2.18-20.85 ; \mathrm{P}=0.00)$ compared with patients in control group. RAP patients with alcohol abuse had an OR of $7.41(95 \% \mathrm{Cl}, 1.8-30.55 ; \mathrm{P}=0.01)$ compared with patients in control group. CP patients with alcohol abuse had an OR of $18.89(95 \% \mathrm{Cl}, 3.84-93 ; \mathrm{P}=0.00)$ compared with patients in control group. RAP patients with type 2 diabetes had an OR of $7.73(95 \% \mathrm{Cl}, 2.08-28.69 ; \mathrm{P}=0.00)$ compared with patients in control group. CP patients with type 2 diabetes had an OR of $6.71(95 \% \mathrm{Cl}, 1.73-26.06 ; \mathrm{P}=0.01)$ compared with patients in control group, besides AP patients with type 2 diabetes had no significant difference compared with control group. RAP patients with heart disease had an OR of $10.15(95 \% \mathrm{Cl}, 1.91-53.82 ; \mathrm{P}=0.01)$ compared with patients in control group. There was no significant association in age, gender, smoking, hypertension, family chronic disease and allergic history between patients with different types of pancreatitis and the control group.

\section{D-dimer, dyslipidemia and TG/HDL-C ratios in different types of pancreatitis}

In different types of pancreatitis, D-dimer levels in AP patients and RAP patients were significantly higher than those in control group ( $p<0.01)$, and there was no significant difference in D-dimer levels between CP group and control group(Table 3 , Figure 2). AP and RAP patients were subdivided into MAP and non-MAP groups (MSAP and SAP groups). For the prediction of severity of AP, the area under curve (AUC) of non-MAP group for serum D-dimer levels was $0.851(p<0.01)$, the cut-off value was $1.13 \mathrm{mg} / \mathrm{dL}$. For the prediction of RAP severity , the AUC of non-MAP group for serum D-dimer levels was $0.80(p<0.05)$, the cut-off value was $2.14 \mathrm{mg} / \mathrm{dL}$ (Table 4, Figure 4).

In this study, the severities of pancreatitis were recognized according to 2012 Atlanta classification, and measured by BISAP score [25], the BISAP scores of AP patients were significantly higher than those of RAP group $(p<0.05)$. The correlation coefficient between the BISAP score and D-dimer level was $0.427(p<0.01)$ (Table 3, Figure 2). Our studies showed that in different types of pancreatitis, the dyslipidemia was significantly different between the AP, RAP group and the control group. Therefore, dyslipidemia in each group was analyzed. Levels of HDL-C in different types of pancreatitis were significantly lower than those in control group but had no significant difference between each pancreatic group. Levels of TG and NEFA were significantly higher in AP and RAP groups than those in control group $(p<0.05)$ but were not significantly different between AP and RAP groups. TG/HDL-C ratios in different types of pancreatitis were significantly higher than those in control group (Table 2, Figure 3). The relationship between D-dimer level and dyslipidemia occurrence was analyzed, and the correlation coefficient between them was $0.33(p<0.01)$ (Table 3). For the prediction of RAP severity, AUC of non-MAP group for TG/HDL-C ratio was 0.84 ( $p<0.05$ ), the cut-off value was 3.51 (Table 4, Figure 4). For the prediction of the relationship between TG/HDL-C and D-dimer level in pancreatitis, the patients were divided into D-dimer elevated group and normal Ddimer group, the AUC of D-dimer elevated group for TG/HDL-C ratio was $0.69(p<0.01)$, the cut-off value was 2.56( $<<0.01)$, in RAP the AUC was 0.73 , and the cut-off value was 2.10 , but there is no significant relationship between D-dimer and TG/HDL$C$ ratio in CP group (Table 4, Figure 4).

\section{Discussion}

\section{Characters of patients with different types of pancreatitis}

RAP refers to a clinical entity characterized by episodes of acute pancreatitis which occurs on more than one occasion. An established chronic disease may be found either on the occasion of the first episode of pancreatitis or during the follow-up. RAP was the strongest predicting factor for a subsequent diagnosis of CP $[4,26,27]$. The etiology and epidemiology of AP, RAP and CP were studied in this research. The results showed that coagulation disorder is related to AP and RAP group, with OR of 13.88 and $12.53(\mathrm{P}<0.01)$ respectively compared with patients in control group, and $\mathrm{D}$-dimer levels in AP and 
RAP groups were significantly higher than those of the control group. However, coagulation disorder and D-dimer levels had no significant difference between CP group and control group. The D-dimer level is also related to the severity of pancreatitis. This result was confirmed in other studies, which showed that D-dimer was an early predictor of the severity of AP [28-30]. The possible mechanism may be two-sided: the abnormal activation of pancreatic enzymes results in inflammation and injury to the pancreas at the onset of AP, which then induces thrombosis and further aggravates the injury[31, 32]. The systemic inflammatory response syndrome and multiple organ dysfunction syndromes, which occur in patients with AP, are common risk factors for the development of VTE [33]. The propagation of the acute inflammatory response can lead to chronic inflammation if there is no appropriate resolution $[34,35]$. On a molecular level, pancreatic stellate cells (PaSCs) have been found to play an important role in models of CP. In CP, PaSCs participate in pathogenesis after transforming into an activated or "myofibroblastic" state [36]. In this myofibroblastic state, PaSCs produce collagen and other extracellular matrix proteins that lead to fibrosis. PaSCs also secrete cytokines which further promote the inflammatory process[36]. However, the D-dimer levels of CP patients were not very different from those of the control group; the reason may be that the severity of inflammation in CP patients was less than that of AP and RAP groups. Besides, the fibrotic changes of the pancreas may not induce acute inflammation and coagulation disturbance. A study about splanchnic venous thrombosis (SVT) suggested that local inflammation plays a major role in SVT causation. Thrombophilia caused by coagulation disturbance is seen in one-third of patients with AP but does not seem to increase the risk of SVT[37].

Our study shows that dyslipidemia is related to different types of AP, RAP and CP, with OR of 8.40, 10.24 and $2.46(p<0.05)$ respectively, and dyslipidemia is also related to D-Dimer level. In previous studies, dyslipidemia, especially hypertriglyceridemia, is related to AP and RAP [38], the pathophysiology could be the metabolism of excessive TGs by pancreatic lipase to free fatty acids (FFA) leading to pancreatic cell injury and ischemia [39]. A 3558-patient study comparing high triglyceride-AP (HTG-AP) versus non-HTG-AP s reported a statistically significant higher incidence of pancreatic necrosis, infected pancreatic necrosis, organ failure, and persistent organ failure [40]. Recent studies demonstrate a trend towards severity in patients with HTG-AP when compared with non-HTG pancreatitis patients; the patients with higher TGs levels appear to have more severe hospital courses with a higher incidence of complications (35-69\%) and organ failure (20-35\%)[41]. Researches have shown that patients with diabetes, obesity, or metabolic syndrome due to insulin resistance tend to have low HDL-C because of lower lipoprotein lipase activity and triglyceride enrichment, but the mechanism is still unknown [42-44]. The TG/HDL-C ratio was found to be associated with insulin resistance in overweight and obese children[45]. Our research shows that in AP and RAP patients, TG and FFA levels of which were significantly higher than those of CP and control group, HDL-C levels of AP, RAP and CP groups were lower than those of the control group $(p<0.05), T G / H D L-C$ ratios of AP, RAP and CP groups were significantly higher than those of the control group $(p<0.05)$, which may be related to insulin resistance in all types of pancreatitis[46, 47]. TG/HDL-C ratio is also a potential useful marker_to identify the severity of RAP patients in clinical practice, with positive predictive value of 0.84 at 3.51 cut-off point. TG/HDL-C ratio was related to insulin resistance, which may occur after acute pancreatitis[48]. In our research, TG/HDL-C ratio is found to be a predictor of severity in RAP patients but not in first attack of AP. The mechanism may be that hypertriglyceridemia (HTG) is a well-established cause of RAP, and TG/HDL-C ratio is predictable in RAP. The relationship between insulin resistance and RAP should be further investigated [39].

We also found that D-dimer level was related to TG/HDL-C ratio $(r=0.379,-p<0.01)$, a previous study has shown that both TG and acute pancreatitis could cause an elevation in d-dimer level, in which TG plays a more important role[49]. In type 2 diabetic children and adolescent, D-dimer level was significantly correlated with TG $(p<0.05)$ [50]. Our research showed that TG was related to D-dimer level in RAP group, the AUC of TG in elevated D-dimer group were less than AUC of TG/HDL-C ratio, the correlation coefficient between TG and D-dimer is less than TG/HDL-C ratio and D-dimer level. So TG/HDL-C could be used as a better predictor for RAP severity and elevated D-dimer level.

Alcoholism is also a risk factor of all types of pancreatitis. Recent data from predominant western countries have shown an increasing trend in the incidence of acute pancreatitis and the number of hospital admissions for both acute and chronic pancreatitis [51-53]. The pathogenesis may be PSCs, which are activated directly by alcohol and its metabolites, and also by 
cytokines and growth factors released during alcohol-induced pancreatic necroinflammation. Activated PSCs are the key cells responsible for fibrosis of alcoholic chronic pancreatitis[54]. Our research shows that alcoholism is related to AP, RAP and CP groups, with OR of $6.74,7.41$ and $18.89(p<0.01)$ respectively.

Our research shows that diabetes is related to RAP and CP. The pathogenesis is that RAP and CP, which are pancreatic inflammation with irreversible parenchymal damage and functional changes, is complicated by progressive nutrient maldigestion, glucose intolerance, diabetes mellitus, and metabolic derangements [55]. Destruction of islet cells by pancreatic inflammation can lead to the development of "brittle" disease with wide swings in blood sugar which are difficult to control. Besides, patients may have pre-existing risk factors for type 2 diabetes, such as insulin resistance, obesity, or dietary habits that further complicate the optimal regulation of glucose metabolism[56-58]. In our study, diabetes was related to RAP and CP groups, with OR of 7.73 and $6.71(p<0.05)$, but had no relationship to the AP group.

In our study, heart disease is related to RAP, with OR of $10.15(\mathrm{p}<0.05)$. So far, research on association between heart and pancreas disease has been paid little attention and its role in pathogenesis is not fully elucidated. The pathogenesis could be pancreatic enzymes and their inhibitors that profoundly affected blood coagulation and appear to influence the course of pancreatic inflammation [59]. Researches have shown that in patients with chronic heart failure, the splanchnic circulation is decreased, especially in highly vascularized pancreas, which may cause pancreatitis [60].

There are no significant differences in other factors like smoking, hypertension, etc._between pancreatitis and the control group. Although we drew similar conclusions from recent studies, our research had significant advantages. We distinguished different types of pancreatitis between AP, RAP and CP groups. RAP is a syndrome of multiple distinct acute inflammatory responses originating within the pancreas in individuals with genetic, environmental, traumatic, morphologic, metabolic, biologic, and/or other risk factors who experienced 2 or more episodes of documented AP, and RAP may lead to chronic pancreatitis [61]. Approximately $9 \%$ to $31 \%$ of patients with AP develop RAP $[62,63]$. To our knowledge, no similar investigations about a comparison between AP, RAP and CP groups have been investigated in China. In our research, the epidemiology and etiological factors of RAP are similar to those of first attack AP; also D-Dimer levels and dyslipidemia were similar in the RAP and the AP groups. However, the D-Dimer level, dyslipidemia and severity of pancreatitis in CP group were similar to those of the control group.

The present research has several limitations. Firstly, it was conducted in a single center with a relatively small sample, which may create bias. Secondly, we did not take into account some inflammation factors, such as PCT, C-reactive protein and interleukin-6; or arterial blood gases, which are also useful predictors of severity in AP[64, 65], because they were not routinely analyzed at admission in Qilu Hospital. Besides, we used BISAP score instead of APACH-『-score to analyze the severity of pancreatitis because some test results needed in the APACH- $₫$ score system were not available, and research had confirmed that BISAP is as useful as APACHE-II and more effective than Ranson criteria, CTSI, CRP, HCT and BMI in predicting severity, organ failure, and death in patients with acute pancreatitis[25]. However, it may still create bias, especially when compared with other studies.

\section{Conclusions}

We confirmed the diagnostic value of D-dimer and TG/HDL-C ratio in predicting the severity of AP and RAP. This suggested that D-dimer might be a useful early predictive biomarker for non-MAP of AP and RAP groups, and TG/HDL-C ratio could be a predictive index for non-MAP of RAP patients, but as the disease progress to $C P$, the value of D-dimer level and TG/HDL-C ratio decreases. The TG/HDL-C ratio is related to D-dimer level in AP and RAP groups. These simple and feasible marker and index could be used in clinical practice to improve early management of AP and RAP, which suggests that the early intervention of coagulation disturbance and dyslipidemia could be substantial. The relationship between D-dimer and dyslipidemia need further investigation.

\section{Material And Methods}


In this observational study, the data of all patients were retrospectively collected from Shandong University Qilu hospital between January 1, 2017, and December 31, 2020. The ethics committee of Qilu Hospital of Shandong University reviewed and approved this study (No.KYLL-202008-096). All the data are anonymous, and the requirement for informed consent was therefore waived. The informed consent waiver statement was approved by the ethics committee of Qilu Hospital of Shandong University. The approval was supported in supplementary material.

\section{Study population}

A review of all medical registries with International Disease_Classification-10 diagnoses related to AP and CP between January 2017 and December 2020 in Shandong University Qilu hospital was performed; RAP patients were selected from AP patients on the basis of RAP definition.

AP was defined as the presence of two or more of the following: (a) abdominal pain compatible with AP, (b) serum amylase and/or lipase values $\geq 3$ times upper limits of normal, (c) imaging findings of AP[66]. RAP was defined as 2 or more welldocumented separate attacks of AP with complete resolution for 3 or more months between attacks according to the definitions provided by INSPPIRE (International Study Group of Pediatric Pancreatitis: in Search for a Cure) [67, 68]. CP patients were selected from patients with CP diagnosis and with CT or MRI exams which were considered consistent with chronic pancreatitis. Normal persons without pancreatitis, malignant diseases, pregnancy, or organ failure, who had health check-ups were enrolled in the control group.

The severities of AP and RAP patients were well recognized according to the latest 2012 revision of the Atlanta classification. Mild AP (MAP) patients were not associated with organ failure (OF) and local or systemic complications. Moderately severe AP (MSAP) was characterized by the presence of transient OF (less than $48 \mathrm{~h}$ ) or local or systemic complications. Severe AP (SAP) was defined as persistent OF for more than $48 \mathrm{~h}$. The diagnosis of OF was based on the modified Marshall scoring system, and a score of 2 or more was considered to be the presence of OF of the respiratory, cardiovascular, or renal systems[69]. BISAP score was used to assess the severity of pancreatitis. Individual components of the BISAP scoring system were shown in Table 1[70].

\section{Data collection}

Age, gender, medical history, and admission number of each patient were collected as baseline demographic data. Moreover, we recorded values of vital signs of all patients on admission and important laboratory parameters. D-dimers were measured in hospital with standard operation procedure; the upper limit of normal value for D-dimer is 0.55 $\mathrm{mg} / \mathrm{L}$. Cholesterol (Cho), high-density lipoprotein cholesterol (HDL-C), low-density lipoprotein cholesterol (LDL-C), and triglyceride (TG) were detected in Department of Laboratory of Qilu Hospital. Prothrombin time (PT), activated partial thromboplastin time (APTT), fibrinogen, and thrombin time were also collected. Values of patients who had 2 or more attacks were counted as the average values of every episode. Patients were considered to have dyslipidemia if total cholesterol (Cho) $>240 \mathrm{mg} / \mathrm{dL}$ or $6.00 \mathrm{mmol} / \mathrm{L}$, high-density lipoprotein cholesterol (HDL-C) $<40 \mathrm{mg} / \mathrm{dL}$ or $0.8 \mathrm{mmol} / \mathrm{L}$, lowdensity lipoprotein cholesterol (LDL-C) $\geq 160 \mathrm{mg} / \mathrm{dL}$ or $3.37 \mathrm{mmol} / \mathrm{L}$, or triglyceride $(T G) \geq 200 \mathrm{mg} / \mathrm{dL}$ or $1.7 \mathrm{mmol} / \mathrm{L}$. Coagulation disorder was recorded if an extension of the PT (>13.8s) or an extension of the APTT (>42s) or D-dimer $>0.55$ $\mathrm{mg} / \mathrm{L}$ occurred. All methods were performed in accordance with the relevant guidelines and regulations.

\section{Statistic analysis}

All statistical analyses were performed using two-tailed test and SPSS version 17.0 and GraphPad Prism v8.0 for Windows (SPSS, Chicago, IL). Data was presented as mean with standard deviation (SD). Comparisons between variables were performed using the one-way ANOVA analysis. For associations between 2 variables, Pearson's chi-square test or Spearman 
rank correlation test was applied, as appropriate. The area under the receiver-operating characteristic (ROC) curve (AUC) was used to assess the predictive accuracy of severity of pancreatitis and to determine the optimum cut-off points with optimal sensitivity and specificity. The AUC was calculated using 95\% confidence interval (Cl). Multivariate logistic regression analysis was adjusted for potential confounding variables, and the odds ratios (OR) and $95 \%$ confidence intervals (Cl) were calculated. $P$ value less than 0.05 was considered statistically significant.

\section{Abbreviations}

$\mathrm{AP}=$ acute pancreatitis, RAP = recurrent acute pancreatitis, $\mathrm{CP}=$ chronic pancreatitis, BISAP = bedside index for severity in acute pancreatitis, AUC = an area under the curve, TG =triglyceride, Cho = cholesterol, LDL-C = low-density lipoprotein cholesterol, HDL-C = high-density lipoprotein cholesterol, FFA = free fatty acid

\section{Declarations}

\section{Acknowledgment}

This work was supported by the Science Foundation of Qilu hospital Shandong University (no. 2015QLMS05)

\section{Author contribution}

Zheng Luo, Xiaoqing Jia contributed to the conception of the study;

Zheng Luo, Xiaoting Zhang, Rong Li, Na Yang performed the data collection, statistical analysis and paper writing;

Xiaoqing Jia, Dalong Sun helped perform the analysis with constructive discussions.

All of the authors have read and approved the final manuscript.

Zheng Luo, Department of Geriatric Medicine, Qilu Hospital of Shandong University

Xiaoting Zhang, Department of Geriatric Medicine, Qilu Hospital of Shandong University

Dalong Sun, Department of Geriatric Medicine, Qilu Hospital of Shandong University

Rong Li, Department of Geriatric Medicine, Qilu Hospital of Shandong University

Na Yang, Department of Geriatric Medicine, Qilu Hospital of Shandong University

Xiaoqing Jia, Department of Gastroenterology and Hepatology, Qilu Hospital of Shandong University

\section{References}

1. Henzen C, Röck M, Schnieper C, Heer K. [Heparin and insulin in the treatment of acute hypertriglyceridemia-induced pancreatitis]. Schweizerische medizinische Wochenschrift 1999;129:1242-1248.

2. Testoni PA. Acute recurrent pancreatitis: Etiopathogenesis, diagnosis and treatment. World journal of gastroenterology 2014;20:16891-16901. 
3. Machicado JD, Yadav D. Epidemiology of Recurrent Acute and Chronic Pancreatitis: Similarities and Differences. Digestive diseases and sciences 2017;62:1683-1691.

4. Lew D, Afghani E, Pandol S. Chronic Pancreatitis: Current Status and Challenges for Prevention and Treatment. Digestive diseases and sciences 2017;62:1702-1712.

5. Kumar S, Ooi CY, Werlin S, Abu-El-Haija M, Barth B, Bellin MD, et al. Risk Factors Associated With Pediatric Acute Recurrent and Chronic Pancreatitis: Lessons From INSPPIRE. JAMA pediatrics 2016;170:562-569.

6. Leppäniemi A, Tolonen M, Tarasconi A, Segovia-Lohse H, Gamberini E, Kirkpatrick AW, et al. 2019 WSES guidelines for the management of severe acute pancreatitis. World J Emerg Surg 2019;14:27-27.

7. Berzigotti A, García-Criado A, Darnell A, García-Pagán JC. Imaging in clinical decision-making for portal vein thrombosis. Nature reviews Gastroenterology \& hepatology 2014;11:308-316.

8. Toqué L, Hamy A, Hamel JF, Cesbron E, Hulo P, Robert S, et al. Predictive factors of splanchnic vein thrombosis in acute pancreatitis: A 6-year single-center experience. Journal of digestive diseases 2015;16:734-740.

9. Maeda K, Hirota M, Ichihara A, Ohmuraya M, Hashimoto D, Sugita H, et al. Applicability of disseminated intravascular coagulation parameters in the assessment of the severity of acute pancreatitis. Pancreas 2006;32:87-92.

10. Feistritzer C, Wiedermann CJ. Effects of anticoagulant strategies on activation of inflammation and coagulation. Expert opinion on biological therapy 2007;7:855-870.

11. Hagiwara S, Iwasaka H, Shingu C, Matsumoto S, Uchida T, Noguchi T. Antithrombin III prevents cerulein-induced acute pancreatitis in rats. Pancreas 2009;38:746-751.

12. Franco-Avilés L, Hernández-Rocha FI, Mercado U, Malvido-Torres CG. [Segmental portal hypertension with splenic vein thrombosis caused by pancreatitis]. Revista medica del Instituto Mexicano del Seguro Social 2017;55:788-790.

13. Li H, Yang Z, Tian F. Clinical Characteristics and Risk Factors for Sinistral Portal Hypertension Associated with Moderate and Severe Acute Pancreatitis: A Seven-Year Single-Center Retrospective Study. Medical science monitor: international medical journal of experimental and clinical research 2019;25:5969-5976.

14. Xu W, Qi X, Chen J, Su C, Guo X. Prevalence of Splanchnic Vein Thrombosis in Pancreatitis: A Systematic Review and Meta-Analysis of Observational Studies. Gastroenterol Res Pract 2015;2015:245460-245460.

15. Weber SM, Rikkers LF. Splenic vein thrombosis and gastrointestinal bleeding in chronic pancreatitis. World journal of surgery 2003;27:1271-1274.

16. Olson JD. D-dimer: An Overview of Hemostasis and Fibrinolysis, Assays, and Clinical Applications. Advances in clinical chemistry 2015;69:1-46.

17. Linkins LA, Takach Lapner S. Review of D-dimer testing: Good, Bad, and Ugly. International journal of laboratory hematology 2017;39 Suppl 1:98-103.

18. Hijazi Z, Oldgren J, Siegbahn A, Wallentin L. Application of Biomarkers for Risk Stratification in Patients with Atrial Fibrillation. Clinical chemistry 2017;63:152-164.

19. Watanabe H, Horita N, Shibata Y, Minegishi S, Ota E, Kaneko T. Diagnostic test accuracy of D-dimer for acute aortic syndrome: systematic review and meta-analysis of 22 studies with 5000 subjects. Scientific reports 2016;6:26893.

20. Triant VA, Lee H, Hadigan C, Grinspoon SK. Increased acute myocardial infarction rates and cardiovascular risk factors among patients with human immunodeficiency virus disease. The Journal of clinical endocrinology and metabolism 2007;92:2506-2512.

21. McLaughlin T, Reaven G, Abbasi F, Lamendola C, Saad M, Waters D, et al. Is there a simple way to identify insulinresistant individuals at increased risk of cardiovascular disease? The American journal of cardiology 2005;96:399-404.

22. Borrayo G, Basurto L, González-Escudero E, Diaz A, Vázquez A, Sánchez L, et al. TG/HDL-C RATIO AS CARDIOMETABOLIC BIOMARKER EVEN IN NORMAL WEIGHT WOMEN. Acta endocrinologica (Bucharest, Romania: 2005) 2018;14:261-267. 
23. Nur Zati Iwani AK, Jalaludin MY. TG: HDL-C Ratio Is a Good Marker to Identify Children Affected by Obesity with Increased Cardiometabolic Risk and Insulin Resistance. 2019;2019:8586167.

24. Xia W, Yao X, Chen Y, Lin J, Vielhauer V, Hu H. Elevated TG/HDL-C and non-HDL-C/HDL-C ratios predict mortality in peritoneal dialysis patients. BMC nephrology 2020;21:324.

25. Hagjer S, Kumar N. Evaluation of the BISAP scoring system in prognostication of acute pancreatitis - A prospective observational study. International journal of surgery (London, England) 2018;54:76-81.

26. Yadav D, O'Connell M, Papachristou GI. Natural history following the first attack of acute pancreatitis. The American journal of gastroenterology 2012;107:1096-1103.

27. Lankisch PG, Breuer N, Bruns A, Weber-Dany B, Lowenfels AB, Maisonneuve P. Natural history of acute pancreatitis: a long-term population-based study. The American journal of gastroenterology 2009;104:2797-2805; quiz 2806.

28. Wan J, Yang X, He W, Zhu Y, Zhu Y, Zeng H, et al. Serum D-dimer levels at admission for prediction of outcomes in acute pancreatitis. 2019;19:67.

29. Zhang GQ, Wang G, Li L, Hu JS, Ji L, Li YL, et al. Plasma D-Dimer Level Is an Early Predictor of Severity of Acute Pancreatitis Based on 2012 Atlanta Classification. Medical science monitor: international medical journal of experimental and clinical research 2019;25:9019-9027.

30. Gomercic C, Gelsi E, Van Gysel D, Frin AC, Ouvrier D, Tonohouan M, et al. Assessment of D-Dimers for the Early Prediction of Complications in Acute Pancreatitis. Pancreas 2016;45:980-985.

31. Kakafika A, Papadopoulos V, Mimidis K, Mikhailidis DP. Coagulation, platelets, and acute pancreatitis. Pancreas 2007;34:15-20.

32. Warzecha Z, Sendur P, Ceranowicz P, Dembinski M, Cieszkowski J, Kusnierz-Cabala B, et al. Pretreatment with low doses of acenocoumarol inhibits the development of acute ischemia/reperfusion-induced pancreatitis. Journal of physiology and pharmacology: an official journal of the Polish Physiological Society 2015;66:731-740.

33. Streiff MB, Agnelli G, Connors JM, Crowther M, Eichinger S, Lopes R, et al. Guidance for the treatment of deep vein thrombosis and pulmonary embolism. Journal of thrombosis and thrombolysis 2016;41:32-67.

34. Habtezion A. Inflammation in acute and chronic pancreatitis. Current opinion in gastroenterology 2015;31:395-399.

35. Gukovsky I, Li N, Todoric J, Gukovskaya A, Karin M. Inflammation, autophagy, and obesity: common features in the pathogenesis of pancreatitis and pancreatic cancer. Gastroenterology 2013;144:1199-1209.e1194.

36. Omary MB, Lugea A, Lowe AW, Pandol SJ. The pancreatic stellate cell: a star on the rise in pancreatic diseases. J Clin Invest 2007;117:50-59.

37. Ahmed SU, Rana SS, Ahluwalia J, Varma N, Sharma R, Gupta R, et al. Role of thrombophilia in splanchnic venous thrombosis in acute pancreatitis. Annals of gastroenterology 2018;31:371-378.

38. Yan $\mathrm{P}$, Zhao H-X, Chen X. Suboptimal management of hypertriglyceridemia in the outpatient setting is associated with the recurrent pancreatitis: A retrospective cohort study. Medicine 2020;99:e22887-e22887.

39. Scherer J, Singh VP, Pitchumoni CS, Yadav D. Issues in hypertriglyceridemic pancreatitis: an update. Journal of clinical gastroenterology 2014;48:195-203.

40. He WH, Zhu Y, Zhu Y, Liu P, Zeng H, Xia L, et al. [Comparison of severity and clinical outcomes between hypertriglyceridemic pancreatitis and acute pancreatitis due to other causes]. Zhonghua yi xue za zhi 2016;96:25692572.

41. Garg R, Rustagi T. Management of Hypertriglyceridemia Induced Acute Pancreatitis. 2018;2018:4721357.

42. Vergès B. Lipid modification in type 2 diabetes: the role of LDL and HDL. Fundamental \& clinical pharmacology 2009;23:681-685.

43. Amigó N, Mallol R, Heras M, Martínez-Hervás S, Blanco Vaca F, Escolà-Gil JC, et al. Lipoprotein hydrophobic core lipids are partially extruded to surface in smaller HDL: "Herniated" HDL, a common feature in diabetes. Scientific reports 2016;6:19249.

Page 10/18 
44. Holmes MV, Millwood IY, Kartsonaki C, Hill MR, Bennett DA, Boxall R, et al. Lipids, Lipoproteins, and Metabolites and Risk of Myocardial Infarction and Stroke. Journal of the American College of Cardiology 2018;71:620-632.

45. Yoo DY, Kang YS, Kwon EB, Yoo EG. The triglyceride-to-high density lipoprotein cholesterol ratio in overweight Korean children and adolescents. 2017;22:158-163.

46. Ko J, Skudder-Hill L, Cho J, Bharmal SH, Petrov MS. The Relationship between Abdominal Fat Phenotypes and Insulin Resistance in Non-Obese Individuals after Acute Pancreatitis. Nutrients 2020;12:2883.

47. Niebisz-Cieślak AB, Karnafel W. Insulin sensitivity in chronic pancreatitis and features of insulin resistance syndrome. Polskie Archiwum Medycyny Wewnetrznej 2010;120:255-263.

48. Ko J, Skudder-Hill L, Cho J, Bharmal SH, Petrov MS. The Relationship between Abdominal Fat Phenotypes and Insulin Resistance in Non-Obese Individuals after Acute Pancreatitis. 2020;12.

49. Kong H, Ding Z, Zhu XC, Gao XY, Wu J, Qian W, et al. d-Dimer change in human acute pancreatitis as determined by serumal triglyceride. Pancreas 2011;40:1103-1106.

50. El Asrar MA, Adly AA, El Hadidy ES, Abdelwahab MA. D-dimer levels in type 1 and type 2 diabetic children and adolescents; Relation to microvascular complications and dyslipidemia "own data and review". Pediatric endocrinology reviews: PER 2012;9:657-668.

51. Lankisch PG, Apte M, Banks PA. Acute pancreatitis. Lancet (London, England) 2015;386:85-96.

52. O'Farrell A, Allwright S, Toomey D, Bedford D, Conlon K. Hospital admission for acute pancreatitis in the Irish population, 1997 2004: could the increase be due to an increase in alcohol-related pancreatitis? Journal of public health (Oxford, England) 2007;29:398-404.

53. Spanier BW, Dijkgraaf MG, Bruno MJ. Trends and forecasts of hospital admissions for acute and chronic pancreatitis in the Netherlands. European journal of gastroenterology \& hepatology 2008;20:653-658.

54. Apte MV, Pirola RC, Wilson JS. Mechanisms of alcoholic pancreatitis. Journal of gastroenterology and hepatology 2010;25:1816-1826.

55. Rickels MR, Bellin M, Toledo FGS, Robertson RP, Andersen DK, Chari ST, et al. Detection, evaluation and treatment of diabetes mellitus in chronic pancreatitis: recommendations from PancreasFest 2012. Pancreatology 2013;13:336-342.

56. Cui Y, Andersen DK. Pancreatogenic diabetes: special considerations for management. Pancreatology 2011;11:279294.

57. Diagnosis and classification of diabetes mellitus. Diabetes care 2013;36 Suppl 1:S67-74.

58. Ewald N, Bretzel RG. Diabetes mellitus secondary to pancreatic diseases (Type 3c)-are we neglecting an important disease? European journal of internal medicine 2013;24:203-206.

59. Nikolic S, Dugic A, Steiner C, Tsolakis AV, Haugen Löfman IM, Löhr JM, et al. Chronic pancreatitis and the heart disease: Still terra incognita? World journal of gastroenterology 2019;25:6561-6570.

60. Nyman LR, Wells KS, Head WS, McCaughey M, Ford E, Brissova M, et al. Real-time, multidimensional in vivo imaging used to investigate blood flow in mouse pancreatic islets. J Clin Invest 2008;118:3790-3797.

61. Guda NM, Muddana V, Whitcomb DC, Levy P, Garg P, Cote G, et al. Recurrent Acute Pancreatitis: International State-ofthe-Science Conference With Recommendations. Pancreas 2018;47:653-666.

62. Gullo L, Migliori M, Pezzilli R, Oláh A, Farkas G, Levy P, et al. An update on recurrent acute pancreatitis: data from five European countries. The American journal of gastroenterology 2002;97:1959-1962.

63. Gao YJ, Li YQ, Wang Q, Li SL, Li GQ, Ma J, et al. Analysis of the clinical features of recurrent acute pancreatitis in China. Journal of gastroenterology 2006;41:681-685.

64. Zhang J, Niu J, Yang J. Interleukin-6, interleukin-8 and interleukin-10 in estimating the severity of acute pancreatitis: an updated meta-analysis. Hepato-gastroenterology 2014;61:215-220.

65. Harrison DA, D'Amico G, Singer M. The Pancreatitis Outcome Prediction (POP) Score: a new prognostic index for patients with severe acute pancreatitis. Critical care medicine 2007;35:1703-1708.

Page 11/18 
66. Bradley EL, 3rd. A clinically based classification system for acute pancreatitis. Summary of the International Symposium on Acute Pancreatitis, Atlanta, Ga, September 11 through 13, 1992. Archives of surgery (Chicago, III: 1960) 1993;128:586-590.

67. Morinville VD, Husain SZ, Bai H, Barth B, Alhosh R, Durie PR, et al. Definitions of pediatric pancreatitis and survey of present clinical practices. Journal of pediatric gastroenterology and nutrition 2012;55:261-265.

68. Yan P, Zhao HX, Chen X. Suboptimal management of hypertriglyceridemia in the outpatient setting is associated with the recurrent pancreatitis: A retrospective cohort study. Medicine 2020;99:e22887.

69. Banks PA, Bollen TL, Dervenis C, Gooszen HG, Johnson CD, Sarr MG, et al. Classification of acute pancreatitis-2012: revision of the Atlanta classification and definitions by international consensus. Gut 2013;62:102-111.

70. Singh VK, Wu BU, Bollen TL, Repas K, Maurer R, Johannes RS, et al. A prospective evaluation of the bedside index for severity in acute pancreatitis score in assessing mortality and intermediate markers of severity in acute pancreatitis. The American journal of gastroenterology 2009;104:966-971.

\section{Tables}

\section{Table 1 \\ BISAP scoring system}

BISAP scoring system

Blood Urea Nitrogen $(B U N)>25 \mathrm{mg} / \mathrm{dl}$

Impaired mental status (Glasgow Coma Scale Score < 15)

Systemic Inflammatory Response Syndrome (SIRS):

SIRS is defined as presence of two or more of the following

1) Temperature of $<36$ or $>38^{\circ} \mathrm{C}$

2) Respiratory rate $>20$ breaths/min or $\mathrm{P}$ a CO2 $<32 \mathrm{~mm} \mathrm{Hg}$

3) Pulse $>90$ beats/min

4) $\mathrm{WBC}<4000$ or $>12,000$ cells $/ \mathrm{mm} 3$ or $>10 \%$ immature bands

Age $>60$ years

Pleural effusion detected on imaging

BISAP: bedside index of severity in acute pancreatitis, WBC: white blood count.

One point is assigned for each variable within $24 \mathrm{~h}$ of presentation and added for a composite score of $0-5$. 
Table 2

Patients' characteristics and predictors by univariate analysis in different types of pancreatitis.

\begin{tabular}{|c|c|c|c|c|c|}
\hline variable & & AP & RAP & $\mathrm{CP}$ & control \\
\hline \multirow[t]{3}{*}{ gender } & female (N\%) & $40.00 \%$ & $27.66 \%$ & $20.97 \%$ & $33.82 \%$ \\
\hline & male (N\%) & $60.00 \%$ & $72.34 \%$ & $79.03 \%$ & $66.18 \%$ \\
\hline & $\mathrm{OR}(95 \% \mathrm{Cl})$ & $0.77(0.40-1.47)$ & $1.35(0.67-2.72)$ & $1.93(0.87-4.25)$ & \\
\hline \multirow[t]{2}{*}{ age } & median age & $50(18-91)$ & $45(22-94)$ & $52.5(30-78)$ & $\begin{array}{l}51(18- \\
79)\end{array}$ \\
\hline & $\mathrm{OR}(95 \% \mathrm{Cl})$ & $1.00(0.97-1.03)$ & $1.00(0.98-1.02)$ & $0.97(0.93-1.01)$ & \\
\hline \multirow[t]{3}{*}{ smoking } & no (N\%) & $64.21 \%$ & $55.32 \%$ & $38.71 \%$ & $77.94 \%$ \\
\hline & yes $(\mathrm{N} \%)$ & $35.79 \%$ & $44.68 \%$ & $61.29 \%$ & $22.06 \%$ \\
\hline & OR & $0.98(0.24-4.10)$ & $2.71(0.39-18.85)$ & $1.07(0.18-6.47)$ & \\
\hline \multirow[t]{3}{*}{ alcoholic abuse } & no (N\%) & $58.95 \%$ & $61.70 \%$ & $32.26 \%$ & $86.76 \%$ \\
\hline & yes (N\%) & $41.05 \%$ & $38.30 \%$ & $67.74 \%$ & $13.24 \%$ \\
\hline & $\mathrm{OR}(95 \% \mathrm{Cl})$ & $\begin{array}{l}5.98(1.48- \\
24.15)^{\star}\end{array}$ & $9.12(1.31-63.6)^{\star}$ & $\begin{array}{l}26.65(4.39- \\
161.67)^{\star \star}\end{array}$ & \\
\hline \multirow[t]{3}{*}{ hypertension } & no (N\%) & $67.37 \%$ & $85.11 \%$ & $69.35 \%$ & $80.88 \%$ \\
\hline & yes $(\mathrm{N} \%)$ & $32.63 \%$ & $14.89 \%$ & $30.65 \%$ & $19.12 \%$ \\
\hline & $\mathrm{OR}(95 \% \mathrm{Cl})$ & $1.70(0.50-5.72)$ & $0.74(0.10-5.15)$ & $1.35(0.32-5.74)$ & \\
\hline \multirow[t]{3}{*}{ heart disease } & no (N\%) & $90.53 \%$ & $82.98 \%$ & $91.94 \%$ & $95.59 \%$ \\
\hline & yes $(\mathrm{N} \%)$ & $9.47 \%$ & $17.02 \%$ & $8.06 \%$ & $4.41 \%$ \\
\hline & $\mathrm{OR}(95 \% \mathrm{Cl})$ & $4.88(0.61-39.26)$ & $\begin{array}{l}16.64(0.58- \\
475.29)\end{array}$ & $10.64(1-113.62)$ & \\
\hline \multirow[t]{3}{*}{ diabetes } & no (N\%) & $86.32 \%$ & $74.47 \%$ & $70.97 \%$ & $92.65 \%$ \\
\hline & yes (N\%) & $13.68 \%$ & $25.53 \%$ & $29.03 \%$ & $7.35 \%$ \\
\hline & $\mathrm{OR}(95 \% \mathrm{Cl})$ & $0.41(0.07-2.40)$ & $2.67(0.29-24.65)$ & $7.36(1.28-42.23) *$ & \\
\hline \multirow[t]{3}{*}{ allergic history } & no (N\%) & $80.00 \%$ & $82.98 \%$ & $90.32 \%$ & $89.71 \%$ \\
\hline & yes $(\mathrm{N} \%)$ & $20.00 \%$ & $17.02 \%$ & $9.68 \%$ & $10.29 \%$ \\
\hline & $\mathrm{OR}(95 \% \mathrm{Cl})$ & $2.95(0.71-12.23)$ & $2.22(0.35-13.89)$ & $0.90(0.15-5.25)$ & \\
\hline \multirow{3}{*}{$\begin{array}{l}\text { family chronic } \\
\text { disease }\end{array}$} & no (N\%) & $94.74 \%$ & $91.49 \%$ & $91.94 \%$ & $98.53 \%$ \\
\hline & yes (N\%) & $5.26 \%$ & $8.51 \%$ & $8.06 \%$ & $1.47 \%$ \\
\hline & $\mathrm{OR}(95 \% \mathrm{Cl})$ & $\begin{array}{l}6.27(0.05- \\
794.39)\end{array}$ & $1.88 \mathrm{E}+08(0--)$ & 4.1E+07 (0--) & \\
\hline \multirow[t]{3}{*}{ dyslipidemia } & no (N\%) & $76.84 \%$ & $80.85 \%$ & $98.39 \%$ & $75.00 \%$ \\
\hline & yes (N\%) & $23.16 \%$ & $19.15 \%$ & $1.61 \%$ & $25.00 \%$ \\
\hline & $\mathrm{OR}(95 \% \mathrm{Cl})$ & $\begin{array}{l}8.40(4.11- \\
17.15)^{\star \star}\end{array}$ & $\begin{array}{l}10.24(4.74- \\
22.11)^{\star \star}\end{array}$ & $2.09(0.63-6.91)$ & \\
\hline
\end{tabular}




\begin{tabular}{|c|c|c|c|c|c|}
\hline \multirow{3}{*}{$\begin{array}{l}\text { coagulation } \\
\text { disorder }\end{array}$} & no (N\%) & $29.47 \%$ & $42.55 \%$ & $74.19 \%$ & $85.29 \%$ \\
\hline & yes $(\mathrm{N} \%)$ & $70.53 \%$ & $57.45 \%$ & $25.81 \%$ & $14.71 \%$ \\
\hline & $\mathrm{OR}(95 \% \mathrm{Cl})$ & $\begin{array}{l}13.88(6.22- \\
30.98)^{\star \star}\end{array}$ & $\begin{array}{l}12.53(5.51- \\
28.49)^{\star *}\end{array}$ & $3.43(0.63-6.91)$ & \\
\hline \multirow[t]{4}{*}{ etiology } & Alcoholic & $15.79 \%$ & $14.89 \%$ & $32.26 \%$ & \\
\hline & Biliary & $64.21 \%$ & $34.04 \%$ & $53.23 \%$ & \\
\hline & Hypertriglyceridemia & $5.26 \%$ & $17.02 \%$ & $0.00 \%$ & \\
\hline & Idiopathic & $14.74 \%$ & $10.64 \%$ & $14.52 \%$ & \\
\hline \multirow[t]{3}{*}{ severity } & MAP & $86.32 \%$ & $93.62 \%$ & & \\
\hline & MSAP & $8.42 \%$ & $4.26 \%$ & & \\
\hline & SAP & $5.26 \%$ & $2.13 \%$ & & \\
\hline
\end{tabular}

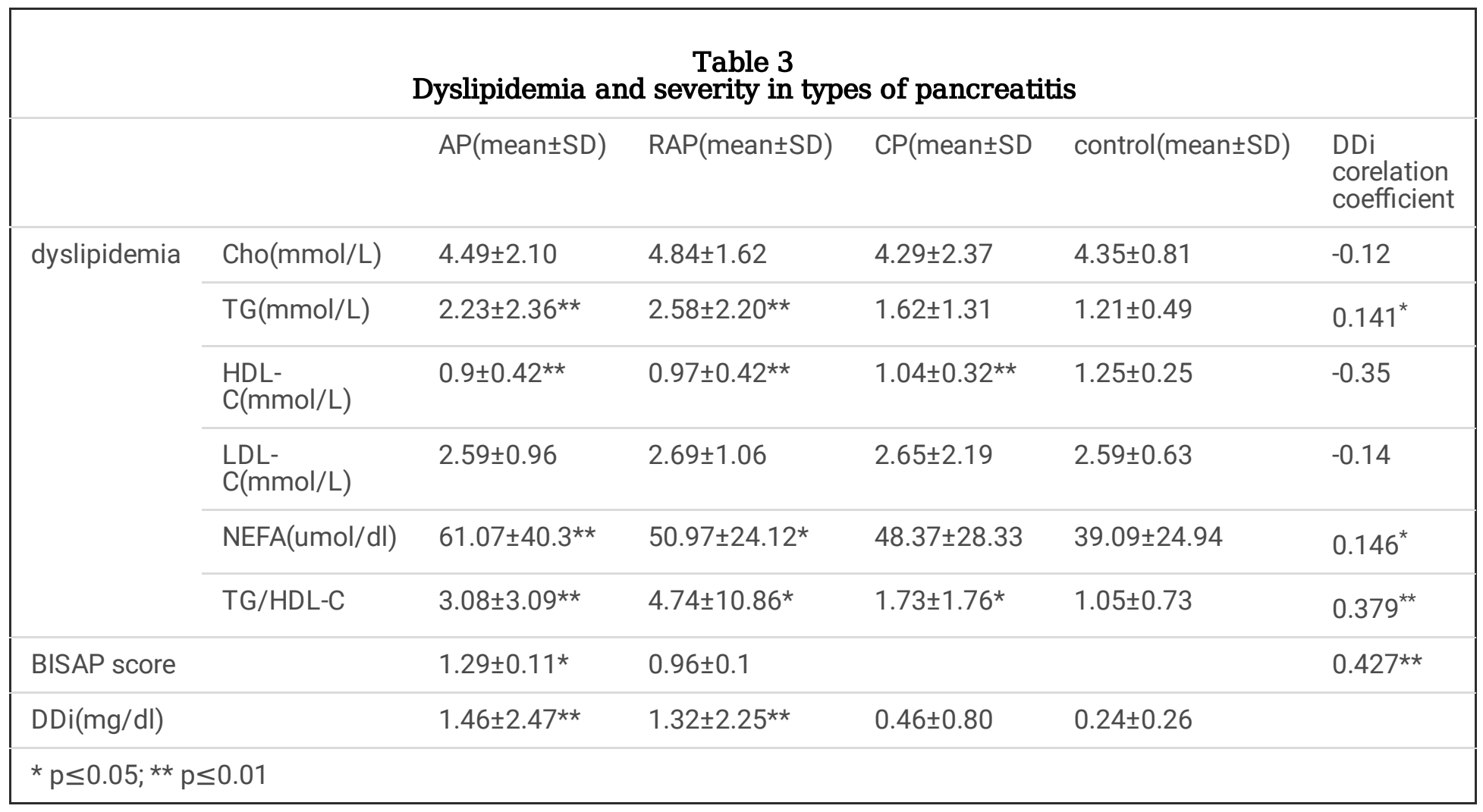

Table 4

ROC cure and cut-off value of D-dimer and dyslipidemia in different types of pancreatitis. 


\begin{tabular}{|c|c|c|c|c|c|c|c|c|c|c|}
\hline \multirow{2}{*}{\multicolumn{2}{|c|}{ Ddi vs severity of pancreatitis\# }} & \multicolumn{3}{|l|}{ AP } & \multicolumn{3}{|l|}{ RAP } & \multicolumn{3}{|l|}{$\mathrm{CP}$} \\
\hline & & \multirow{2}{*}{$\begin{array}{l}\text { AUC } \\
0.85\end{array}$} & \multirow{2}{*}{$\begin{array}{l}\begin{array}{l}\text { cut-off } \\
\text { vaue }\end{array} \\
1.13\end{array}$} & \multirow{2}{*}{$\begin{array}{l}\mathrm{p} \\
0.00\end{array}$} & \multirow{2}{*}{$\begin{array}{l}\text { AUC } \\
0.80\end{array}$} & \multirow{2}{*}{$\begin{array}{l}\begin{array}{l}\text { cut-off } \\
\text { vaue }\end{array} \\
2.14\end{array}$} & \multirow{2}{*}{$\begin{array}{l}\mathrm{p} \\
0.04\end{array}$} & \multirow[t]{2}{*}{ AUC } & \multirow{2}{*}{$\begin{array}{l}\text { cut- } \\
\text { off } \\
\text { vaue }\end{array}$} & \multirow[t]{2}{*}{$\mathrm{p}$} \\
\hline & & & & & & & & & & \\
\hline \multirow{6}{*}{$\begin{array}{l}\text { dyslipidemia vs severity } \\
\text { of pancreatitis }\end{array}$} & Cho & 0.17 & - & 0.00 & 0.21 & 2.22 & 0.05 & & & \\
\hline & TG & 0.50 & 1.39 & 0.97 & 0.63 & 2.09 & 0.39 & & & \\
\hline & HDL-C & 0.25 & - & 0.00 & 0.08 & 0.22 & 0.00 & & & \\
\hline & LDL-C & 0.14 & 4.05 & 0.00 & 0.34 & 1.42 & 0.29 & & & \\
\hline & NEFA & 0.46 & 237.00 & 0.65 & 0.28 & 10.50 & 0.14 & & & \\
\hline & $\begin{array}{l}\text { TG/HDL- } \\
\mathrm{C}\end{array}$ & 0.61 & 2.17 & 0.22 & 0.84 & 3.51 & 0.02 & & & \\
\hline \multirow[t]{6}{*}{ dyslipidemia vs Ddi } & Cho & 0.36 & 6.42 & 0.02 & 0.50 & 5.98 & 0.98 & 0.46 & 3.33 & 0.65 \\
\hline & TG & 0.61 & 1.37 & 0.08 & 0.70 & 2.17 & 0.00 & 0.58 & 1.18 & 0.37 \\
\hline & HDL-C & 0.26 & 2.39 & 0.00 & 0.36 & 1.49 & 0.03 & 0.23 & - & 0.00 \\
\hline & LDL-C & 0.36 & 3.86 & 0.02 & 0.48 & 3.52 & 0.74 & 0.52 & 1.86 & 0.83 \\
\hline & NEFA & 0.45 & 40.50 & 0.46 & 0.55 & 42.50 & 0.44 & 0.75 & 46.50 & 0.00 \\
\hline & $\begin{array}{l}\text { TG/HDL- } \\
\mathrm{C}\end{array}$ & 0.69 & 2.56 & 0.00 & 0.73 & 2.10 & 0.00 & 0.67 & 2.31 & 0.05 \\
\hline
\end{tabular}

\# the severity of pancreatitis was subdivided into MAP and non-MAP groups.

Figures 


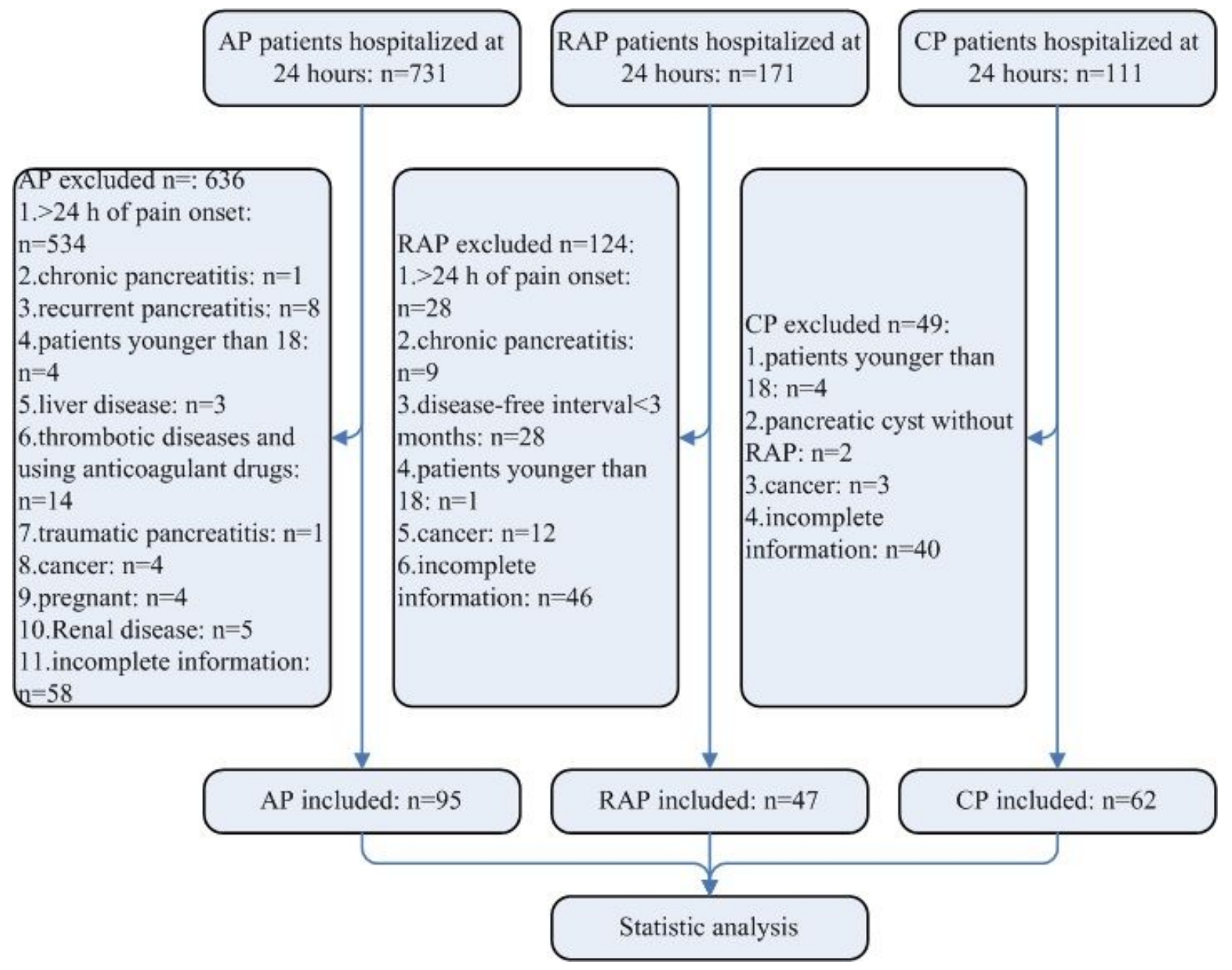

Figure 1

Flow chart of the study
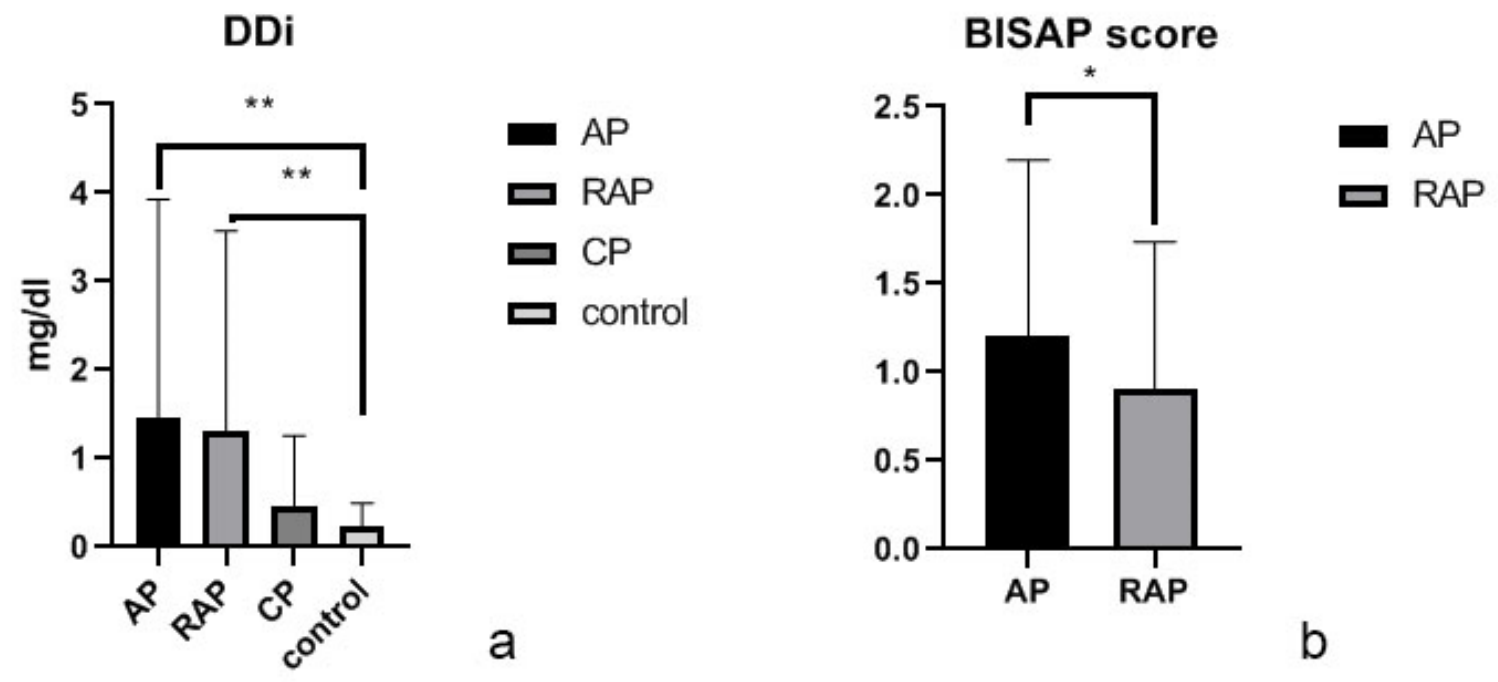

Figure 2 
D-dimer level in different types of pancreatitis and severity of different types of pancreatitis: a. D-dimer expression in types of pancreatitis. b. BISAP score in different types of pancreatitis. ${ }^{* *} \mathrm{p} \leq 0.01$
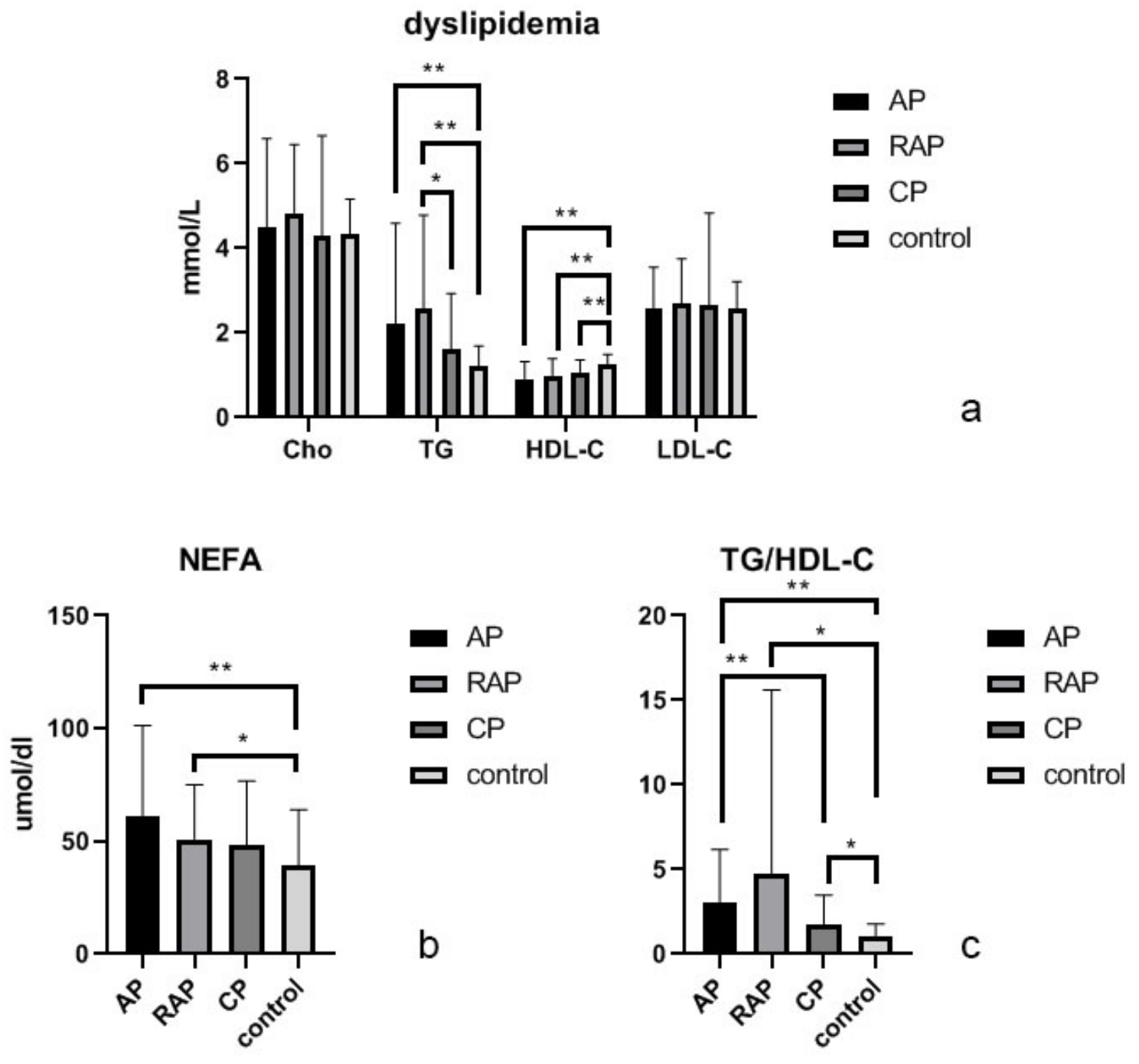

Figure 3

Dyslipidemia in different types of pancreatitis. a. Dyslipidemia in different types of pancreatitis; $b$. Free fatty acid in different types of pancreatitis; c. TG/HDL-C ratio in different types of pancreatitis. ${ }^{*} p \leq 0.05 ;{ }^{* \star} p \leq 0.01$

Figure 4

ROC curves of D-dimer and dyslipidemia in different types of pancreatitis. a. ROC curves for D-dimer level to distinguish MAP and non-MAP group in AP. b. ROC curves for D-dimer level to distinguish MAP and non-MAP group in RAP. c. ROC curves for D-dimer level to distinguish MAP and non-MAP group. d. ROC curves for TG/HDL-C level to distinguish MAP and non-MAP group in AP. e. ROC curves for TG/HDL-C level to distinguish MAP and non-MAP group in RAP. f. ROC curves for TG/HDL-C level to distinguish MAP and non-MAP group. g. ROC curves for TG/HDL-C level to distinguish normal D-dimer 
group and increased D-dimer group in AP. h. ROC curves for TG/HDL-C level to distinguish normal D-dimer group and increased D-dimer group in RAP. i. ROC curves for TG/HDL-C level to distinguish normal D-dimer group and increased Ddimer group in $C P .{ }^{*} p \leq 0.05$; ${ }^{* *} p \leq 0.01$ 